\title{
A Note on the Structure of Roller Coaster Permutations
}

\author{
William Adamczak ${ }^{1}$ \\ ${ }^{1}$ Department of Mathematics, Siena College, Loudonville, NY, USA \\ Correspondence: William Adamczak, Department of Mathematics, Siena College, Loudonville, NY, USA. E-mail: wadam- \\ czak@siena.edu
}

Received: February 28, 2017 Accepted: March 21, 2017 Online Published: May 25, 2017

doi:10.5539/jmr.v9n3p75

URL: https://doi.org/10.5539/jmr.v9n3p75

\begin{abstract}
In this paper we consider the structure of a special class of permutations known as roller coaster permutations, first introduced by Ahmed \& Snevily (2013). A roller coaster permutation is described as, a permutation that maximizes the total switches from ascending to descending, or visa versa, for the permutation as well as all of its subpermutations, simultaneously. This paper looks at the structure of these permutations, particularly the alternating structure, what the entires of these permutations can look like, we then introduce a notion of a condition stronger than alternating that we shall refer to as recursively alternating.
\end{abstract}

Keywords: permutations, combinatorics

\section{Introduction}

The idea of roller coaster permutations first shows up in a work of Ahmed \& Snevily (2013), where they are described as permutations that maximize the total switches from ascending to descending, or visa versa, for the permutation and all of its subpermutations simultaneously. More basically, this permutation gets the greatest number of ups and downs for the permutation and all possible subpermutations simultaneously.

These permutations have strong relations to pattern avoiding permutations as do permutations that are merely alternating permutations, as seen in Mansour (2004) in the context of avoiding the subpermutation 132. The connection with forbidden subsequences and partitions of permutations is seen in Stankova (1994), where certain forbidden subsequences end up being roller coaster permutations, particularly $F(1,1)$ is a subset of $R C(n)$. Consequently, these permutations are related to stack sortable permutations as seen in (Egge \& Mansour, 2004), where the connection between forbidden subsequences and stack sortability is made.

\section{Background}

Throughout this paper we will use one-line notation for permutations. Let $[n]=\{1,2, \ldots, n\}$. A permutation $\pi$ will be viewed as a sequence $\left(\pi_{1}, \pi_{2}, \ldots, \pi_{n}\right)$ where the commas may be omitted for small $n$. Let $S_{n}$ denote the set of all permutations of $[n]$.

Definition 1. We will use the following definitions given by Ahmed E Snevily (2013):

$$
\begin{aligned}
& i(\pi)=\text { the number of increasing sequences of contiguous numbers in } \pi, \\
& d(\pi)=\text { the number of decreasing sequences of contiguous numbers in } \pi, \\
& i d(\pi)=i(\pi)+d(\pi), \\
& X(\pi)=\{\tau: \tau \text { is a subsequence of } \pi \text { such that }|\tau| \geq 3\}, \\
& t(\pi)=\sum_{\tau \in X(\pi)} i d(\tau) .
\end{aligned}
$$

Here we refer to contiguous numbers as consisting of at least two numbers. 
Example 2. Consider the permutation 3412 in $S_{4}$ where we have

$$
\begin{aligned}
& X(1324)=\{1324,132,134,412\} \\
& t(1324)=i d(1324)+i d(132)+i d(134)+i d(412)=3+2+1+2=8 .
\end{aligned}
$$

Definition 3. Let $R C(n)=\left\{\pi \in S_{n}: t(\pi)=\max _{\sigma \in S_{n}} t(\sigma)\right\}$.

The members of $R C(n)$ are then referred to as a Roller Coaster Permutations.

Example 4. For $n=3,4,5,6$ we have:

$$
\begin{aligned}
& R C(3)=\{132,213,231,312\} \\
& R C(4)=\{2143,2413,3142,3412\} \\
& R C(5)=\{24153,25143,31524,32514,34152,35142,41523,42513\} \\
& R C(6)=\{326154,351624,426153,451623\}
\end{aligned}
$$

For a more extensive listing see (Ahmed E Snevily, 2013).

Definition 5. For $\pi \in S_{n}$ we will let $\pi[i, j]$ denote the restriction of $\pi$ to indices $i$ through $j$ where $1 \leq i<j \leq n$. We will also use the notation $\pi /\{i, j\}$ to denote the restriction of $\pi$ to all indices other than $i$ and $j$.

\section{Structure of Roller Coaster Permutations}

Ahmed \& Snevily (2013) conjectured that all roller coaster permutations are either alternating or reverse-alternating where they defined these as $\pi_{1}<\pi_{2}>\pi_{3}<\ldots$ and $\pi_{1}>\pi_{2}<\pi_{3}>\ldots$ respectively. Where there is no confusion we will simply refer to such a permutation as alternating, regardless of whether it is alternating or reverse alternating.

Theorem 1. Let $\pi \in R C(n)$, then $\pi$ is an alternating permutation.

Proof. Consider the rightmost positions $i-1, i, i+1$ such that $\pi[i, n]$ is alternating. We may assume these are two consecutive increases, i.e. $\pi_{i}<\pi_{j}$, the case for two consecutive decreases will follow analogously. Consider $\tilde{\pi}=\pi \cdot(i, i+1)$, ie exchanging positions $i$ and $i+1$. Our strategy throughout this proof is to examine the subsequences of $\sigma$ of $\pi$ and $\tilde{\sigma}$ of $\tilde{\pi}$ and to look the relationship between $i d(\sigma)$ and $i d(\tilde{\sigma})$. We may do this based on how they contribute to the total sums $t(\pi)$ and $t(\tilde{\pi})$.

First note that subsequences $\sigma$ of $\pi /\{i, i+1\}$ are identical to the corresponding subsequences of $\tilde{\pi} /\{i, i+1\}$, thus we have $i d(\sigma)=i d(\tilde{\sigma})$.

Note that for the cases where only one of the positions $i$ or $i+1$ is involved in $\sigma$, we will have the same in $\tilde{\sigma}$, since these are merely swapped and as such still show up as subpermutations. Alternately said, note that $\pi /\{i\}=\tilde{\pi} /\{i+1\}$ and $\pi /\{i+1\}=\tilde{\pi} /\{i\}$. We may therefore consider only subpermutations containing these two consecutive positions. For any such subpermutation, $\sigma$, let positions $j-1, j, j+1, j+2$ be such that $\sigma_{j}=\tilde{\pi}_{i}$ and $\sigma_{j+1}=\tilde{\pi}_{i+1}$.

Now note that the worst case is to have a subpermutation $\sigma$ such that $\sigma_{j-1}>\sigma_{j}>\sigma_{j+1}>\sigma_{j+1}$, as this would decrease the ascent/descent count by two. Note that $\sigma_{j-1}$ cannot be $\pi_{i-1}$ since $\pi_{i-1}<\pi_{i}$ by assumption and $\pi_{i-1}<\pi_{i+1}$. Now note that for every subsequence $\sigma$, not involving $\pi_{i}$, there is a correspondence subsequence involving $\pi_{i}$. Those without may decrease the ascent/descent count by at most two in $t(\tilde{\pi})$ as compared to in $t(\pi)$, however any subsequence involving $\pi_{i}$ will certainly increase the ascent/descent count by two in $t(\tilde{\pi})$, so at worst these subsequences will not increase the total count, but this cannot decrease.

Now observe that we have not yet accounted for the subsequence involving only positions $i$ through $i+2$, which sees an increase in the ascent/descent count by one as we compare $t(\tilde{\pi})$ to $t(\pi)$, thus the total count must increase, implying that the total ascent/descent count is greater for $\tilde{\pi}$ than for $\pi$, ie $t(\tilde{\pi})>t(\pi)$, which contradicts the assumption that $\pi$ was in $R C(n)$.

The next property of note, regarding roller coaster permutations, is that the value in the first and final positions of the permutation must differ by exactly one. This will be used in a later proof involving a deeper self-similar structure of roller coaster permutations.

Theorem 2. Let $\pi \in R C(n)$, then $\left|\pi_{n}-\pi_{1}\right|=1$, i.e. the starting and ending entry differ by exactly one. 
Proof. Suppose that $\pi$ does not satisfy this condition. Then there exists an $i, 1<i<n$, such that either $\pi_{1}<\pi_{i}<\pi_{n}$ or $\pi_{1}>\pi_{i}>\pi_{n}$. Without loss of generality we may assume $\pi_{1}<\pi_{i}<\pi_{n}$ since the reverse is merely an operation on the reverse of the permuation which is of course also in $R C(n)$.

Pick $i$ the be the position corresponding to the largest value of $\pi_{i}$ such that the inequality $\pi_{1}<\pi_{i}<\pi_{n}$ holds. Then consider the permutation $\pi^{\prime}=\pi \cdot(i, n)$. We claim that $t\left(\pi^{\prime}\right)>t(\pi)$ which would contradict the assumption that $\pi \in R C(n)$.

Note that, for $1<j \neq i<n$, we have that either $\pi_{j}<\pi_{i}, \pi_{n}$ or $\pi_{j}>\pi_{i}, \pi_{n}$. Thus, for any subsequence other than $\pi_{1}, \pi_{i}, \pi_{n}$, the total number of ascents and descents remains unchanged since the inequalities relative to the positions remain unchanged otherwise. Note however that the subsequence $\pi_{1}^{\prime}, \pi_{i}^{\prime}, \pi_{n}^{\prime}$ increases the count of ascents and descents by one, thereby establishing the claim.

We would next like to observe how the values in odd positions and the values in even positions occur in roller coaster permutations.

Theorem 3. Let $\pi \in R C(n)$. If $\pi$ is an alternating (not reverse-alternating) permutation, then $\pi_{i}>\pi_{1}, \pi_{n}$ for $i$ even and $\pi_{i}<\pi_{1}, \pi_{n}$ for $i$ odd. As well, if $\pi$ is a reverse-alternating permutation, then $\pi_{i}<\pi_{1}, \pi_{n}$ for $i$ even and $\pi_{i}>\pi_{1}, \pi_{n}$ for $i$ odd.

Proof. We will show this in the context of an alternating permutation in $R C(n)$ as the proof for reverse-alternating is analogous.

Begin by finding the leftmost indices $j, j+1$ where both $\pi_{j}$ and $\pi_{j+1}$ are greater than the maximum of $\pi_{1}$ and $\pi_{n}$. Note that $\pi_{j}>\pi_{j+1}$ by the choice of the leftmost pair. Also note that by alternating we have that $\pi_{j+2}$ is also greater than the maximum of $\pi_{1}$ and $\pi_{n}$.

Let $\sigma=\pi(l, r)$ where the index $l$ is such that $\pi_{l}=\max \left\{\pi_{i} \mid 1 \leq i<j, \pi_{1}, \pi_{n} \leq \pi_{i}<\pi_{j}\right\}$. Then observe that $t(\sigma)>t(\pi)$ via an argument analogous to that used in proving that these permutations are alternating since the index $l$ selected for make the exchange is chosen precisely to avoid interfering with subsequences not involving the exchanged positions. This contradicts that $\pi \in R C(n)$.

To simplify the idea, this is basically stating that all the odd positioned entires have values that are all greater than the start and ending value of the permutation or all less than, and the reverse for the even positioned entries. Based on this result we quickly get major insights into the structure of these permutations. Breaking these into the cases where $n$ is odd/even and the permutation is alternating versus reverse-alternating will add additional structural insight which follows easily from the results to this point.

Corollary 4. For $\pi \in R C(n)$ with $n$ odd and $\pi$ an alternating permutation we have that:

- $\left\{\pi_{1}, \pi_{n}\right\}=\left\{\frac{n-1}{2}, \frac{n+1}{2}\right\}$

- $\left\{\pi_{j} \mid 3 \leq j \leq n-2, j\right.$ odd $\}=\left\{1, \ldots, \frac{n-3}{2}\right\}$

- $\left\{\pi_{j} \mid 2 \leq j \leq n-1, j\right.$ even $\}=\left\{\frac{n+3}{2}, \ldots, n\right\}$

For $\pi \in R C(n)$ with $n$ odd and $\pi$ a reverse-alternating permutation we have that:

- $\left\{\pi_{1}, \pi_{n}\right\}=\left\{\frac{n+1}{2}, \frac{n+3}{2}\right\}$

- $\left\{\pi_{j} \mid 2 \leq j \leq n-1, j\right.$ odd $\}=\left\{\frac{n+5}{2}, \ldots, n\right\}$

- $\left\{\pi_{j} \mid 3 \leq j \leq n-2, j\right.$ even $\}=\left\{1, \ldots, \frac{n-1}{2}\right\}$

For $\pi \in R C(n)$ with $n$ even and $\pi$ an alternating permutation we have that:

- $\left\{\pi_{1}, \pi_{n}\right\}=\left\{\frac{n}{2}, \frac{n}{2}+1\right\}$

- $\left\{\pi_{j} \mid 3 \leq j \leq n-2, j\right.$ odd $\}=\left\{1, \ldots, \frac{n}{2}-1\right\}$

- $\left\{\pi_{j} \mid 2 \leq j \leq n-1, j\right.$ even $\}=\left\{\frac{n}{2}+2, \ldots, n\right\}$ 
For $\pi \in R C(n)$ with $n$ even and $\pi$ a reverse-alternating permutation we have that:

$$
\begin{array}{ll}
\text { - } & \left\{\pi_{1}, \pi_{n}\right\}=\left\{\frac{n}{2}, \frac{n+2}{2}\right\} \\
\text { - } & \left\{\pi_{j} \mid 2 \leq j \leq n-1, j \text { odd }\right\}=\left\{\frac{n}{2}+2, \ldots, n\right\} \\
\text { - } & \left\{\pi_{j} \mid 3 \leq j \leq n-2, j \text { even }\right\}=\left\{1, \ldots, \frac{n}{2}-1\right\}
\end{array}
$$

Proof. Merely observe that the previous result together with the fact that the start and end of the permutation differ by one gives that all values greater than the start and end are either in even or odd positions depending on whether this is alternating or reverse-alternating.

Note here, that for $n$ odd, a permutation and its reverse will be both alternating or both reverse-alternating. When $n$ is even this is no longer the case, which starts to lend some insight into how different the order of $R C(n)$ is in the odd versus even cases.

While we have seen that roller coaster permutations are in fact alternating, we will prove a stronger condition that we shall refer to as recursively alternating. We will continue to use the notation $\pi[i, j]$ to refer to the restriction of the permutation $\pi$ to positions $i$ through $j$.

Definition 5. First define $\pi_{\text {odd }}$ as the restriction to the odd indexed positions of $\pi[2, n-1]$ and $\pi_{\text {even }}$ as the restriction to the even indexed positions of $\pi[2, n-1]$.

A permutation $\pi$ is then said to be recursively alternating if:

- $\pi$ is alternating or reverse alternating.

- $\pi_{\text {odd }}$ and $\pi_{\text {even }}$ are alternating or reverse alternating.

- Recursively iterating this process yields alternating or reverse alternating, ie for $\pi_{\text {odd,odd }}$ and $\pi_{\text {odd,even }}$, etc.

We may note that this definition is equivalent to the restriction of $\pi[2, n-1]$ to indices that are congruent modulo $2^{k}$ for some $k$, is alternating.

Theorem 6. If $\pi \in R C(n)$ then $\pi$ is recursively alternating.

Proof. Assume $\pi \in R C(n)$ and is therefore alternating or reverse-alternating. Consider $\pi_{\text {odd }}$ and assume $\pi_{o d d}$ is not alternating or reverse-alternating as the $\pi_{\text {even }}$ case is analogous. Apply the same argument used to show that roller coaster permutations were alternating noting that this modification to $\pi_{\text {odd }}$ does not effect the direction of inequalities relative to other positions of $\pi$ since all values in odd positions are either all greater than or all less than the values of all even positions. We may then iterate this argument as required.

\section{A Quick Example}

We end with a final visual example of a roller coaster permutation, in order that that the discovered properties may be seen explicitly. The permutation $\{4,3,7,1,8,2,6,5\}$, pictured below, is an example of a roller coaster permutation from $R C(8)$. This graph is arrived at is by assigning the $x$ coordinate to the indices and the $y$ coordinate to the entries.

From this visual depiction, the alternating behavior is evident. Note also that we see the same alternating behavior when considering only positions 2,4 , and 6 and we see similar when considering only positions 3,5 , and 7 . This is to be expected based on the noted recursively alternating behavior. Furthermore, we can readily see in this example how all of the odd indices, when excluding the start and end, all correspond to the entries with values greater than 5. Similarly the even indices have the values less than 4 as proven earlier. 


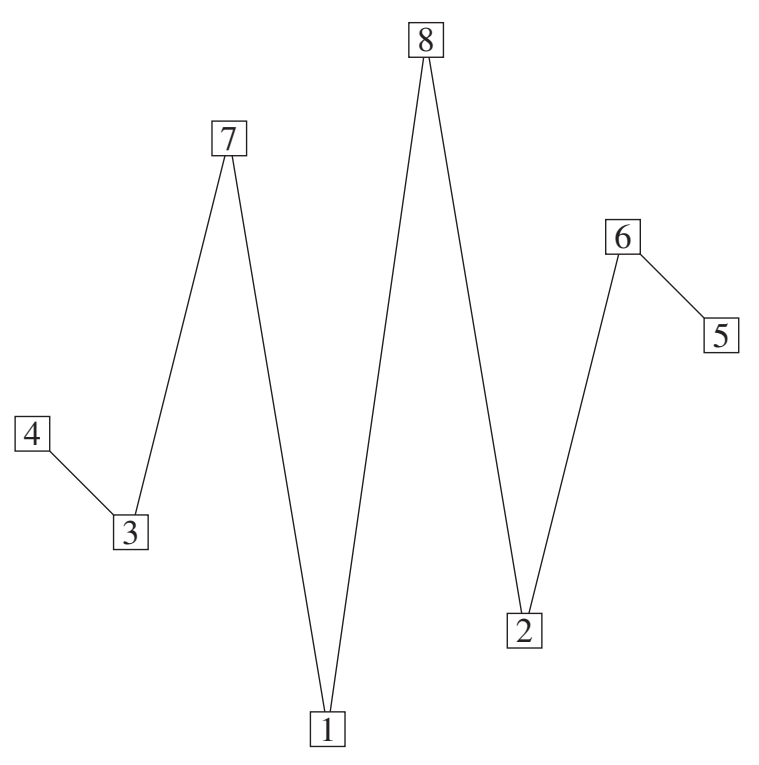

\section{References}

E. Egge, T. Mansour. (2004). 132-avoiding Two-stack Sortable Permutations, Fibonacci Numbers, and Pell Numbers. Discrete Applied Mathematics, 143, 78-83. https://doi.org/10.1016/j.dam.2003.12.007

T. Ahmed, H. Snevily. (2013). Some Properties of Roller Coaster Permutations. Bulletin of the ICA 68, 55-60.

Toufik Mansour. (2003). Restricted 132-alternating permutations and Chebyshev polynomials. Annals of Combinatorics, 7, 201-227. https://doi.org/10.1007/s00026-003-0182-2

Z. E. Stankova. (1994). Forbidden subsequences. Discrete Math., 132, 291-316.

https://doi.org/10.1016/0012-365x(94)90242-9

\section{Copyrights}

Copyright for this article is retained by the author(s), with first publication rights granted to the journal.

This is an open-access article distributed under the terms and conditions of the Creative Commons Attribution license (http://creativecommons.org/licenses/by/4.0/). 